\title{
NOVEL FAULT DETECTION CRITERIA BASED ON LINEAR QUADRATIC CONTROL PERFORMANCES
}

\author{
DUŠAN KROKAVEC, ANNA FILASOVÁ \\ Department of Cybernetics and Artificial Intelligence, Faculty of Electrical Engineering and Informatics \\ Technical University of Košice, Letná 9/B, 04200 Košice, Slovakia \\ e-mail: \{dusan.krokavec, anna.filasova\}@tuke.sk
}

\begin{abstract}
This paper proposes a new approach to designing a relatively simple algorithmic fault detection system that is potentially applicable in embedded diagnostic structures. The method blends the LQ control principle with checking and evaluating unavoidable degradation in the sequence of discrete-time LQ control performance index values due to faults in actuators, sensors or system dynamics. Design conditions are derived, and direct computational forms of the algorithms are given. A simulation example subject to different types of failures is used to illustrate the design process and to demonstrate the effectiveness of the method.
\end{abstract}

Keywords: performance degradation, fault detection schemes, discrete-time systems, LQ control methods.

\section{Introduction}

Automated diagnosis has been one of the more fruitful applications in sophisticated control systems, with potential significance for domains in which systems diagnosis must proceed while the system is operative and testing opportunities are limited by operational considerations. A real problem is usually to fix the system with faults so that it can continue its mission for some time with some limitations in functionality. Consequently, diagnosis is a part of a larger problem known as Fault Detection, Identification and Reconfiguration (FDIR) (Khelassi et al., 2011). The practical benefits of an integrated approach to FDIR seem to be considerable, especially when knowledge of available fault isolations and system reconfigurations is used to reduce the cost and increase the reliability and utility of control and diagnosis. Classical principles include observer-based methods, parity space methods and parameter identification based methods, which have been thoroughly studied (see, e.g., the works of Blanke et al. (2003), Chen and Patton (1999), Korbicz et al. (2004), Simani et al. (2003), and the references therein).

In the last years many significant results have spurred interest in passivity theory, which has been widely used in stability analysis and control of nonlinear systems (e.g., Brogliato et al., 2007; Chen et al., 2011). The physical meaning of the passivity is that the energy stored in the passive systems cannot be greater than the energy supplied by the environment outside. This energy relation can be expressed by an inequality with dependence on the system state, input and output, and by checking this inequality, when it fails, faults in the system are detected. The passivity based fault detection method was extended to the energy based framework (Chen et al., 2010), where, besides the stored and supplied energy, the dissipated energy is modeled for the dissipative systems, and an optimal fault detection system based on the energy balance was suggested.

The problem of controlling a system in such a way as to optimize a performance index that represents the actual operating system performance has been the area of study for several decades (Kirk, 1970; Bryson and Ho, 1975; Anderson and Moore, 1989; Lewis and Syrmos, 1995). In particular, if attention is restricted to Linear Quadratic (LQ) control, several works following this approach over the years have been reported in the literature, with some recent ones (e.g., Dorf and Bishop, 2011; Hendricks et al., 2008) for continuous and discrete-time systems. Specifically, this approach has been often assumed in diverse practical problems for a finite-time interval with time-varying feedback gains and full state measurable variables, to bring dynamical systems to desired final states, with special interest in aircraft, spacecraft, robot control and diagnosis (Henry, 2010; Zolghadri, 2000; Zolghadri et al., 2006). 
Following the given ideas in LQ control performance (Krokavec, 2002), a fault detection scheme based on LQ control properties for a full state measurable case and an infinite time horizon is suggested in the paper. If the LQ control problem is solvable, then a degradation in the sequence of instantaneous values of the LQ performance index is evaluated. A direct form of the algorithms is proposed with the goal to verify, by LQ control, optimized closed-loop parameter relations. Based on discrete-time system state control, as well as on the nominal LQ performance index, generalized fault residual functions are proposed, associated with the standard form of the discrete LQ control criterium. Note that the aim is not to design an LQ control law, but to exploit LQ control characteristics in fault detection if LQ control is given.

The rest of the paper is organized as follows. Starting with system basic preliminaries concerning system models and the LQ control task in Section 2 in Section 3 the basic concept and overall structures, focused on the dissipative property, the forced mode and fault residual functions, are presented. Section 4 shows an example to illustrate fault detection characteristics, and Section 5 is devoted to a brief overview of the properties of the proposed method.

\section{Basic preliminaries}

2.1. System models. Consider a discrete-time linear deterministic dynamic system under normal operating conditions described by the set of equations

$$
\begin{aligned}
\boldsymbol{q}(i+1) & =\boldsymbol{F} \boldsymbol{q}(i)+\boldsymbol{G u}(i), \\
\boldsymbol{y}(i) & =\boldsymbol{C} \boldsymbol{q}(i),
\end{aligned}
$$

where $\boldsymbol{q}(i) \in \mathbb{R}^{n}$ represents the system state, $\boldsymbol{u}(i) \in \mathbb{R}^{r}$ denotes the control input, $\boldsymbol{y}(i) \in \mathbb{R}^{m}$ is the reference output, matrices $\boldsymbol{F} \in \mathbb{R}^{n \times n}, \boldsymbol{G} \in \mathbb{R}^{n \times r}, \boldsymbol{C} \in \mathbb{R}^{m \times n}$ are finite valued, and $i \in \mathbb{Z}_{+}$.

When a fault occurs, the faulty system is given by

$$
\begin{aligned}
\boldsymbol{q}_{f}(i+1) & =\boldsymbol{F}_{f} \boldsymbol{q}_{f}(i)+\boldsymbol{G} \boldsymbol{u}_{f}(i), \\
\boldsymbol{y}_{f}(i) & =\boldsymbol{C} \boldsymbol{q}_{f}(i)
\end{aligned}
$$

where by a system fault the system properties were changed, described now by the matrix $\boldsymbol{F}_{f} \in \mathbb{R}^{n \times n}$. A single sensor fault, as well as a single actuator fault, is represented by the relations

$$
\begin{aligned}
& \boldsymbol{q}_{f}(i)=\left(\boldsymbol{I}_{m}-\boldsymbol{I}_{f s}\right) \boldsymbol{q}(i), \\
& \boldsymbol{u}_{f}(i)=\left(\boldsymbol{I}_{r}-\boldsymbol{I}_{f a}\right) \boldsymbol{u}(i),
\end{aligned}
$$

where $\boldsymbol{I}_{n} \in \mathbb{R}^{n \times n}, \boldsymbol{I}_{r} \in \mathbb{R}^{r \times r}$ are the identity matrices, and $\boldsymbol{I}_{f s} \in \mathbb{R}^{n \times n}, \boldsymbol{I}_{f a} \in \mathbb{R}^{r \times r}$ generally take the structure

$$
\boldsymbol{I}_{f s}, \boldsymbol{I}_{f a} \cong \operatorname{diag}\left[\begin{array}{lllllll}
0 & \cdots & 0 & 1 & 0 & \cdots & 0
\end{array}\right] .
$$

A single parameter fault of the system matrix is represented as

$$
\boldsymbol{F}_{f}=\boldsymbol{F}-\boldsymbol{f}_{f k}^{T} \Delta F_{k h} \boldsymbol{f}_{f h},
$$

where $\Delta F_{k h}$ is an unacceptable change in the system matrix element $F_{k h}$ due to the system parameter fault, and $\boldsymbol{f}_{f k}, \boldsymbol{f}_{f h} \in \mathbb{R}^{n}$ takes the structure

$$
\boldsymbol{f}_{f k}, \boldsymbol{f}_{f a} \cong\left[\begin{array}{lllllll}
0 & \cdots & 0 & 1 & 0 & \cdots & 0
\end{array}\right] .
$$

It is assumed throughout the paper that all state variables are measurable and, to consider the infinite-time control horizon, the couple $(\boldsymbol{F}, \boldsymbol{G})$ is controllable.

2.2. LQ control task. In order to build up the background of the proposed method, some basics on the discrete-time LQ control are recalled. Since the control design is now posed as an optimal problem with some combined quadratic performance on $\boldsymbol{q}(i)$ and $\boldsymbol{u}(i)$, the task is formulated as follows: Find a nonzero control $\boldsymbol{u}(i)$ defined on $\langle 0, N-1\rangle$ such that the state $\boldsymbol{q}(i)$ is driven to the state coordinate origin at $i=N$, and the following performance index is minimized:

$$
J_{N}=\boldsymbol{q}^{T}(N) \boldsymbol{Q}^{\bullet} \boldsymbol{q}(N)+\sum_{i=0}^{N-1} r(\boldsymbol{q}(i), \boldsymbol{u}(i)),
$$

where

$$
\begin{aligned}
r( & \boldsymbol{q}(i), \boldsymbol{u}(i)) \\
= & \boldsymbol{u}^{T}(i) \boldsymbol{R} \boldsymbol{u}(i)+\boldsymbol{q}^{T}(i) \boldsymbol{Q} \boldsymbol{q}(i)+\boldsymbol{q}^{T}(i) \boldsymbol{S} \boldsymbol{u}(i) \\
& +\boldsymbol{u}^{T}(i) \boldsymbol{S}^{T} \boldsymbol{q}(i) \\
= & {\left[\begin{array}{ll}
\boldsymbol{q}^{T}(i) & \boldsymbol{u}^{T}(i)
\end{array}\right] \boldsymbol{J}_{J}\left[\begin{array}{c}
\boldsymbol{q}(i) \\
\boldsymbol{u}(i)
\end{array}\right], }
\end{aligned}
$$

$\boldsymbol{J}_{J} \in \mathbb{R}^{(n+r) \times(n+r)}$ takes the form

$$
\boldsymbol{J}_{J}=\left[\begin{array}{cc}
\boldsymbol{Q} & \boldsymbol{S} \\
\boldsymbol{S}^{T} & \boldsymbol{R}
\end{array}\right]>0 \Leftrightarrow \boldsymbol{Q}-\boldsymbol{S R}^{-1} \boldsymbol{S}^{T}>0,
$$

$N$ is finite, $\boldsymbol{Q} \geq 0, \boldsymbol{Q} \in \mathbb{R}^{n \times n}, \boldsymbol{R}>0, \boldsymbol{R} \in \mathbb{R}^{m \times m}$, $S \in \mathbb{R}^{n \times r}$, and $\boldsymbol{Q}^{\bullet} \geq 0, \boldsymbol{Q}^{\bullet} \in \mathbb{R}^{n \times n}$.

Proposition 1. (Equivalent performance index) If the system (17), (2) is controllable, then the LQ control design task is optimized with respect to the equivalent quadratic cost function (performance index)

$$
J_{N}=\boldsymbol{q}^{T}(0) \boldsymbol{P}(0) \boldsymbol{q}(0)+\sum_{i=0}^{N-1} p(\boldsymbol{q}(i), \boldsymbol{u}(i)),
$$

where

$$
\begin{gathered}
p(\boldsymbol{q}(i), \boldsymbol{u}(i))=\left[\begin{array}{ll}
\boldsymbol{q}^{T}(i) & \boldsymbol{u}^{T}(i)
\end{array}\right] \boldsymbol{J}(i)\left[\begin{array}{c}
\boldsymbol{q}(i) \\
\boldsymbol{u}(i)
\end{array}\right] \\
\boldsymbol{J}(i)=\left[\begin{array}{cc}
\boldsymbol{F}^{T} \boldsymbol{P}(i) \boldsymbol{F}-\boldsymbol{P}(i-1)+\boldsymbol{Q} & \boldsymbol{F}^{T} \boldsymbol{P}(i) \boldsymbol{G}+\boldsymbol{S} \\
\left(\boldsymbol{F}^{T} \boldsymbol{P}(i) \boldsymbol{G}+\boldsymbol{S}\right)^{T} & \boldsymbol{G}^{T} \boldsymbol{P}(i) \boldsymbol{G}+\boldsymbol{R}
\end{array}\right]
\end{gathered}
$$


$\boldsymbol{P}(i)>0, \boldsymbol{P}(i) \in \mathbb{R}^{n \times n}$, and $\boldsymbol{J}(i) \geq 0, \boldsymbol{J}(i) \in$ $\mathbb{R}^{(n+r) \times(n+r)}$.

Proof. (See, e.g., Krokavec, 2002) Since the system (1), (2) is linear in $\boldsymbol{q}(i)$, the quadratic Lyapunov function can be chosen as

$$
v(\boldsymbol{q}(i))=\boldsymbol{q}^{T}(i) \boldsymbol{P}(i-1) \boldsymbol{q}(i),
$$

where $\boldsymbol{P}(-1)=\boldsymbol{P}(0)$. Thus, the increment in the Lyapunov function takes form

$$
\begin{aligned}
& \Delta v(\boldsymbol{q}(i), \boldsymbol{u}(i)) \\
& \quad=v(\boldsymbol{q}(i+1))-v(\boldsymbol{q}(i)), \\
& \quad=\left[\boldsymbol{q}^{T}(i) \boldsymbol{u}^{T}(i)\right] \boldsymbol{J}_{V}(i)\left[\begin{array}{r}
\boldsymbol{q}(i) \\
\boldsymbol{u}(i)
\end{array}\right],
\end{aligned}
$$

where

$$
\boldsymbol{J}_{V}(i)=\left[\begin{array}{cc}
\boldsymbol{F}^{T} \boldsymbol{P}(i) \boldsymbol{F}-\boldsymbol{P}(i-1) & \boldsymbol{F}^{T} \boldsymbol{P}(i) \boldsymbol{G} \\
\left(\boldsymbol{F}^{T} \boldsymbol{P}(i) \boldsymbol{G}\right)^{T} & \boldsymbol{G}^{T} \boldsymbol{P}(i) \boldsymbol{G}
\end{array}\right] .
$$

Defining, at the time instant $N$, the cumulative function $V_{N}$ as

$$
V_{N}=\sum_{i=0}^{N-1} \Delta v(\boldsymbol{q}(i), \boldsymbol{u}(i))
$$

which, in turn, is equivalent to

$$
V_{N}=\boldsymbol{q}^{T}(N) \boldsymbol{P}(N-1) \boldsymbol{q}(N)-\boldsymbol{q}^{T}(0) \boldsymbol{P}(0) \boldsymbol{q}(0),
$$

then adding (19) to (10), subtracting (20) from (10), and setting $\boldsymbol{P}(N-1)=\boldsymbol{Q}^{\bullet}$, the performance index is brought to the form (13), where

$$
p(\boldsymbol{q}(i), \boldsymbol{u}(i))=r(\boldsymbol{q}(i), \boldsymbol{u}(i))+\Delta v(\boldsymbol{q}(i), \boldsymbol{u}(i)) .
$$

It is evident that with $\boldsymbol{J}(i)=\boldsymbol{J}_{J}+\boldsymbol{J}_{V}(i)$, (12) and (18) imply 15.

Proposition 2. (Infinite horizon LQ control) The LQ control law for which the gain has a constant value is given by

$$
\begin{aligned}
\boldsymbol{u}(i) & =-\boldsymbol{K} \boldsymbol{q}(i) \\
\boldsymbol{K} & =\left(\boldsymbol{G}^{T} \boldsymbol{P} \boldsymbol{G}+\boldsymbol{R}\right)^{-1}\left(\boldsymbol{F}^{T} \boldsymbol{P} \boldsymbol{G}+\boldsymbol{S}\right)^{T}
\end{aligned}
$$

where $\boldsymbol{P}>0$ is a solution of the Discrete Algebraic Riccati Equation (DARE)

$$
\begin{aligned}
\boldsymbol{P}= & \boldsymbol{F}^{T} \boldsymbol{P} \boldsymbol{F}+\boldsymbol{Q}-\left(\boldsymbol{F}^{T} \boldsymbol{P} \boldsymbol{G}+\boldsymbol{S}\right)\left(\boldsymbol{G}^{T} \boldsymbol{P} \boldsymbol{G}+\boldsymbol{R}\right)^{-1} \\
& \times\left(\boldsymbol{F}^{T} \boldsymbol{P} \boldsymbol{G}+\boldsymbol{S}\right)^{T} .
\end{aligned}
$$

Proof. (Krokavec, 2002) Considering $\boldsymbol{P}(i)=\boldsymbol{P}, \boldsymbol{J}(i)=$ $\boldsymbol{J}$, (14) and (15) imply

$$
\begin{aligned}
& \frac{\partial p(\boldsymbol{q}(i), \boldsymbol{u}(i))}{\partial \boldsymbol{u}^{T}(i)} \\
& =\left[\begin{array}{ll}
\mathbf{0} & \boldsymbol{I}
\end{array}\right] \boldsymbol{J}\left[\begin{array}{c}
\boldsymbol{q}(i) \\
\boldsymbol{u}(i)
\end{array}\right] \\
& =\left[\begin{array}{ll}
\left(\boldsymbol{F}^{T} \boldsymbol{P} \boldsymbol{G}+\boldsymbol{S}\right)^{T} & \boldsymbol{G}^{T} \boldsymbol{P} \boldsymbol{G}+\boldsymbol{R}
\end{array}\right]\left[\begin{array}{c}
\boldsymbol{q}(i) \\
\boldsymbol{u}(i)
\end{array}\right]=\mathbf{0}, \\
& \frac{\partial p(\boldsymbol{q}(i), \boldsymbol{u}(i))}{\partial \boldsymbol{q}^{T}(i)} \\
& =\left[\begin{array}{ll}
\boldsymbol{I} & \mathbf{0}
\end{array}\right] \boldsymbol{J}\left[\begin{array}{c}
\boldsymbol{q}(i) \\
\boldsymbol{u}(i)
\end{array}\right] \\
& =\left(\boldsymbol{F}^{T} \boldsymbol{P} \boldsymbol{F}-\boldsymbol{P}+\boldsymbol{Q}\right) \boldsymbol{q}(i)+\left(\boldsymbol{F}^{T} \boldsymbol{P} \boldsymbol{G}+\boldsymbol{S}\right) \boldsymbol{u}(i)=\mathbf{0},
\end{aligned}
$$

respectively, where $\boldsymbol{I} \in \mathbb{R}^{n \times n}$ is the identity matrix. It is obvious that (25) implies (23), and by substituting (22) and (23) into (26), Eqn. 24) is obtained.

Moreover, the existence of 24 is conditioned by the inequality

$$
\boldsymbol{G}^{T} \boldsymbol{P} \boldsymbol{G}+\boldsymbol{R}>0,
$$

and the steady-state solution of 15 is

$$
\boldsymbol{J}=\left[\begin{array}{cc}
\boldsymbol{F}^{T} \boldsymbol{P} \boldsymbol{F}-\boldsymbol{P}+\boldsymbol{Q} & \boldsymbol{F}^{T} \boldsymbol{P} \boldsymbol{G}+\boldsymbol{S} \\
\boldsymbol{G}^{T} \boldsymbol{P} \boldsymbol{F}+\boldsymbol{S}^{T} & \boldsymbol{G}^{T} \boldsymbol{P} \boldsymbol{G}+\boldsymbol{R}
\end{array}\right]>0 .
$$

Note that it makes no practical sense to have a terminal cost term with the terminal time being infinite. It should be mentioned that in the sequel only the infinite horizon LQ control of the discrete-time system (1), (2) is considered.

2.3. Tuning of the performance index matrices. Few approaches have been suggested for the selection of the performance index matrices $\boldsymbol{Q}, \boldsymbol{R}$ and $\boldsymbol{S}$. In general, the matrix $Q$ determines the relative importance of the state variables, $\boldsymbol{R}$ represents the expenditure of control signal energy to be restricted, and $\boldsymbol{S}$ characterizes the dissipation of the closed-loop system. Since different values of these matrices will eventually end up with a different system response, LQ control design is often combined with closed-loop pole assignment in a prescribed disc (Furuta and Kim, 1987; Krokavec and Filasová, 2008). Especially the weighting matrices can be set naturally as $\boldsymbol{Q}=\boldsymbol{C}^{T} \boldsymbol{C}, \boldsymbol{R}=\boldsymbol{B}^{T} \boldsymbol{B}$, respectively.

In the book devoted to this case, Bryson (1999) suggested to choose diagonal weighting matrices $Q$ and $\boldsymbol{R}$ with diagonal elements equal to the inverses of the 
desired maximum squared values of variables of $\boldsymbol{q}(t)$ and $\boldsymbol{u}(t)$, respectively, and tuning these iteratively. On the other hand, Anderson and Moore (1989) proposed to search for appropriate $\boldsymbol{Q}$ and $\boldsymbol{R}$ depending on the required bandwidth. Another technique was proposed by Muhafzan (2010).

Completing (11) to the square gives

$$
\begin{aligned}
r(\boldsymbol{q}(i), \boldsymbol{u}(i)) \\
=\left(\boldsymbol{u}^{T}(i)+\boldsymbol{R}^{-1} \boldsymbol{S}^{T} \boldsymbol{q}(i)\right)^{T} \boldsymbol{R}\left(\boldsymbol{u}^{T}(i)\right. \\
\left.\quad+\boldsymbol{R}^{-1} \boldsymbol{S}^{T} \boldsymbol{q}(i)\right)+\boldsymbol{q}^{T}(i)\left(\boldsymbol{Q}-\boldsymbol{S}^{T} \boldsymbol{R}^{-1} \boldsymbol{S}\right) \boldsymbol{q}(i),
\end{aligned}
$$

i.e., for nonzero $S$ the state vector weight of the equivalent system is given by the Schur complement of (12).

\section{Basic concept and overall structures}

3.1. Dissipative property. In dynamical system diagnosis it may be useful to develop state-space or input-output models so that they reflect the dissipativity of the system, and to show how these results can be exploited in fault diagnosis design. The approach used in the paper is based on the next dissipative system properties.

Definition 1. (Brogliato et al., 2007) The discrete time system (1), (2) is said to be dissipative with respect to the supply rate $s(\boldsymbol{y}(h), \boldsymbol{u}(h))$ if there exists a so-called storage function $v(\boldsymbol{q}(i))>0$ such that

$$
v(\boldsymbol{q}(i)) \leq v(\boldsymbol{q}(0))+\sum_{h=0}^{i} s(\boldsymbol{y}(h), \boldsymbol{u}(h)),
$$

along all possible trajectories of the system starting at $\boldsymbol{q}(0)$, for all $\boldsymbol{q}(0), i \geq 0$.

Note that storage functions are often used as Lyapunov functions for studying the stability of an equilibrium of the system with zero input $\boldsymbol{u}(i)$ (unforced regime), and for linear systems dissipativeness is usually considered for quadratic supply rates $s(\boldsymbol{q}(h), \boldsymbol{u}(h))$. Moreover, one of the key properties of a dissipative dynamical system is that the total energy stored in the system decreases with time.

Theorem 1. The system (17), (2) under the LQ control (22) with $\boldsymbol{K}$ satisfying (23), (24) is dissipative in a steadystate with respect to the supply rate $s(\boldsymbol{q}(i), \boldsymbol{u}(i))$ and the storage function $v(\boldsymbol{q}(i))$, where

$$
\begin{aligned}
s(\boldsymbol{q}(i), \boldsymbol{u}(i)) & =\boldsymbol{q}^{T}(i) \boldsymbol{S u}(i), \\
v(\boldsymbol{q}(i)) & =\frac{1}{2} \boldsymbol{q}^{T}(i) \boldsymbol{P} \boldsymbol{q}(i) .
\end{aligned}
$$

Proof. Based on the steady-state value of (14) and (28), we get

$$
p(\boldsymbol{q}(i), \boldsymbol{u}(i))=\boldsymbol{q}^{T}(i)\left[\boldsymbol{I}-\boldsymbol{K}^{T}\right] \boldsymbol{J}\left[\begin{array}{c}
\boldsymbol{I} \\
-\boldsymbol{K}
\end{array}\right] \boldsymbol{q}(i),
$$

and with some straightforward calculations over (33) it can be shown that

$$
\begin{aligned}
& p(\boldsymbol{q}(i), \boldsymbol{u}(i)) \\
& =\boldsymbol{q}^{T}(i)\left\{\begin{array}{c}
\boldsymbol{Q}+\boldsymbol{F}^{T} \boldsymbol{P} \boldsymbol{F}-\boldsymbol{P}- \\
-\left(\boldsymbol{S}+\boldsymbol{F}^{T} \boldsymbol{P} \boldsymbol{G}\right) \boldsymbol{K}- \\
-\boldsymbol{K}^{T}\left(\boldsymbol{S}+\boldsymbol{F}^{T} \boldsymbol{P} \boldsymbol{G}\right)^{T}+ \\
+\boldsymbol{K}^{T}\left(\boldsymbol{R}+\boldsymbol{G}^{T} \boldsymbol{P} \boldsymbol{G}\right) \boldsymbol{K}
\end{array}\right\} \boldsymbol{q}(i) .
\end{aligned}
$$

Since (26) implies that the sum of the first two rows of (34) is equal to zero, by selection of $\boldsymbol{K}$ as in (23) it is easy to see that

$$
\begin{aligned}
& p(\boldsymbol{q}(i), \boldsymbol{u}(i)) \\
& =\boldsymbol{q}^{T}(i) \boldsymbol{K}^{T}\left\{\begin{array}{c}
-\left(\boldsymbol{S}+\boldsymbol{F}^{T} \boldsymbol{P} \boldsymbol{G}\right)^{T}+ \\
+\left(\boldsymbol{R}+\boldsymbol{G}^{T} \boldsymbol{P} \boldsymbol{G}\right) \boldsymbol{K}
\end{array}\right\} \boldsymbol{q}(i) \\
& =\boldsymbol{q}^{T}(i) \boldsymbol{K}^{T}\left\{\begin{array}{c}
-\left(\boldsymbol{S}+\boldsymbol{F}^{T} \boldsymbol{P} \boldsymbol{G}\right)^{T}+ \\
+\left(\boldsymbol{S}+\boldsymbol{F}^{T} \boldsymbol{P} \boldsymbol{G}\right)^{T}
\end{array}\right\} \boldsymbol{K} \boldsymbol{q}(i)=0 .
\end{aligned}
$$

Now, according to (21) and with (35), at a steady state this gives

$$
\Delta v(\boldsymbol{q}(i), \boldsymbol{u}(i))+r(\boldsymbol{q}(i), \boldsymbol{u}(i))=0 .
$$

To obtain an expression for the dissipative property, the summation of (36) from 0 to $k-1$ can be carried out, and with respect to the Lyapunov stability condition this yields

$$
\begin{aligned}
v(\boldsymbol{q}(i)) & -v(\boldsymbol{q}(0))+\sum_{i=0}^{k-1} \boldsymbol{q}^{T}(i) \boldsymbol{S u}(i) \\
+ & \frac{1}{2} \sum_{i=0}^{k-1}\left(\boldsymbol{q}^{T}(i) \boldsymbol{Q} \boldsymbol{q}(i)+\boldsymbol{u}^{T}(i) \boldsymbol{R} \boldsymbol{u}(i)\right) \leq 0 .
\end{aligned}
$$

Thus

$$
v(\boldsymbol{q}(i))-v(\boldsymbol{q}(0)) \leq-\sum_{i=0}^{k-1} \boldsymbol{q}^{T}(i) \boldsymbol{S u}(i),
$$

and, consequently,

$$
\frac{1}{2} \sum_{i=0}^{k-1}\left(\boldsymbol{q}^{T}(i) \boldsymbol{Q} \boldsymbol{q}(i)+\boldsymbol{u}^{T}(i) \boldsymbol{R} \boldsymbol{u}(i)\right) \geq 0 .
$$

Note that the quadratic supply rates $s(\boldsymbol{q}(h), \boldsymbol{u}(h))$ used slightly differ from $s(\boldsymbol{y}(h), \boldsymbol{u}(h))$, usually considered in nonlinear dissipative systems analysis (Haddad and Chellaboina, 2003; Khalil, 2002).

3.2. Forced regime. In practice, the case with $r=$ $=m$ (square plants) is often encountered, where with each output signal a reference signal, is generally associated which is expected to influence this output as desired. Such a regime, reflecting nonzero set working points, is called the forced regime. 
Definition 2. The forced regime for (1), (2) is given by the control policy

$$
\boldsymbol{u}(i)=-\boldsymbol{K} \boldsymbol{q}(i)+\boldsymbol{W} \boldsymbol{w}(i),
$$

where $r=m, \boldsymbol{w}(i) \in \mathbb{R}^{m}$ is the desired output signal vector, and $\boldsymbol{W} \in \mathbb{R}^{m \times m}$ is the signal gain matrix.

Lemma 1. If the system (1), (2) is stabilizable and

$$
\operatorname{rank}\left[\begin{array}{cc}
\boldsymbol{F} & \boldsymbol{G} \\
\boldsymbol{C} & \mathbf{0}
\end{array}\right]=n+m,
$$

then the matrix $\boldsymbol{W}$ in (41), designed by using the static decoupling principle, takes the form

$$
\boldsymbol{W}=\left(\boldsymbol{C}\left(\boldsymbol{I}_{n}-(\boldsymbol{F}-\boldsymbol{G} \boldsymbol{K})\right)^{-1} \boldsymbol{G}\right)^{-1} .
$$

Proof. In a steady state, which corresponds to $\boldsymbol{q}(i+1)=$ $=\boldsymbol{q}(i)=\boldsymbol{q}_{\circ}$, the equality $\boldsymbol{y}_{\circ}=\boldsymbol{w}_{\circ}$ must hold, where $\boldsymbol{q}_{\circ} \in \mathbb{R}^{n}, \boldsymbol{y}_{\circ}, \boldsymbol{w}_{\circ} \in \mathbb{R}^{m}$ are vectors of steady state values of $\boldsymbol{q}(i), \boldsymbol{y}(i), \boldsymbol{w}(i)$, respectively. In this case, (1), (2), (40) can be written as

$$
\begin{aligned}
\mathbf{0} & =-\left(\boldsymbol{I}_{n}-(\boldsymbol{F}-\boldsymbol{G} \boldsymbol{K})\right) \boldsymbol{q}_{\circ}+\boldsymbol{G} \boldsymbol{W} \boldsymbol{w}_{\circ}, \\
\boldsymbol{y}_{\circ} & =\boldsymbol{C} \boldsymbol{q}_{\circ} \\
& =\boldsymbol{C}\left(\boldsymbol{I}_{n}-(\boldsymbol{F}-\boldsymbol{G} \boldsymbol{K})\right)^{-1} \boldsymbol{G} \boldsymbol{W} \boldsymbol{w}_{\circ}=\boldsymbol{I}_{m} \boldsymbol{w}_{\circ},
\end{aligned}
$$

respectively. Thus, (44) implies (42).

Remark 1. The $\boldsymbol{W}$ matrix is nothing else than the inverse of the closed-loop static gain matrix. This gain matrix can be obtained by setting $z=1$ in the state-space expression of the transfer function matrix of the closed-loop system. Note that the static gain realized by the $\boldsymbol{W}$ matrix is ideal in control only if the plant parameters, on which the value of $\boldsymbol{W}$ depends, are perfectly known and do not vary with time.

Remark 2. The forced regime is basically designed for constant references and is very closely related to the shift of origin. If the command value $\boldsymbol{w}(i)$ is changed "slowly enough," the above scheme can do a reasonable job of tracking, i.e., making $\boldsymbol{y}(i)$ follow $\boldsymbol{w}(i)$ (Kailath, 1980).

Lemma 2. Considering a steady-state solution $\boldsymbol{P}$ of (24) and the system (17), (2) in the forced regime with the control policy (22), (23), the performance index density (14) is

$$
p_{w}(\boldsymbol{w}(i))=\boldsymbol{w}^{T}(i) \boldsymbol{W}^{T}\left(\boldsymbol{G}^{T} \boldsymbol{P} \boldsymbol{G}+\boldsymbol{R}\right) \boldsymbol{W} \boldsymbol{w}(i) .
$$

Proof. Inserting (40) into the steady-state value of (14) yields

$$
\begin{aligned}
p(\boldsymbol{q}(i), \boldsymbol{w}(i)) \\
=\left\{\boldsymbol{w}^{T}(i)\left[\mathbf{0} \boldsymbol{W}^{T}\right]+\boldsymbol{q}^{T}(i)\left[\boldsymbol{I}-\boldsymbol{K}^{T}\right]\right\} \\
\quad \times \boldsymbol{J}\left\{\left[\begin{array}{c}
\boldsymbol{I} \\
-\boldsymbol{K}
\end{array}\right] \boldsymbol{q}(i)+\left[\begin{array}{c}
\mathbf{0} \\
\boldsymbol{W}
\end{array}\right] \boldsymbol{w}(i)\right\} .
\end{aligned}
$$

Thus, writing the block partition structure of (28) as

$$
=\left[\begin{array}{cc}
\boldsymbol{F}^{T} \boldsymbol{P} \boldsymbol{F}-\boldsymbol{P}+\boldsymbol{Q} & \boldsymbol{F}^{T} \boldsymbol{P} \boldsymbol{G}+\boldsymbol{S} \\
\boldsymbol{G}^{T} \boldsymbol{P} \boldsymbol{F}+\boldsymbol{S}^{T} & \boldsymbol{G}^{T} \boldsymbol{P} \boldsymbol{G}+\boldsymbol{R}
\end{array}\right]=\left[\begin{array}{ll}
\boldsymbol{J}_{11} & \boldsymbol{J}_{12} \\
\boldsymbol{J}_{12}^{T} & \boldsymbol{J}_{22}
\end{array}\right]
$$

and taking into account that at a steady state the condition (36) implies $p(\boldsymbol{q}(i), \boldsymbol{u}(i))=0$, we have

$$
\begin{aligned}
& p(\boldsymbol{q}(i), \boldsymbol{w}(i))-p(\boldsymbol{q}(i), \boldsymbol{u}(i)) \\
& =p_{w}(\boldsymbol{q}(i), \boldsymbol{w}(i)) \\
& =\left[\begin{array}{c}
\boldsymbol{q}(i) \\
\boldsymbol{W} \boldsymbol{w}(i)
\end{array}\right]^{T}\left[\begin{array}{cc}
\mathbf{0} & \boldsymbol{J}_{12}-\boldsymbol{K}^{T} \boldsymbol{J}_{22} \\
\boldsymbol{J}_{12}^{T}-\boldsymbol{J}_{22} \boldsymbol{K} & \boldsymbol{J}_{22}
\end{array}\right]\left[\begin{array}{c}
\boldsymbol{q}(i) \\
\boldsymbol{W} \boldsymbol{w}(i)
\end{array}\right] .
\end{aligned}
$$

Since (23) gives

$$
\boldsymbol{K}=\left(\boldsymbol{G}^{T} \boldsymbol{P} \boldsymbol{G}+\boldsymbol{R}\right)^{-1}\left(\boldsymbol{F}^{T} \boldsymbol{P} \boldsymbol{G}+\boldsymbol{S}\right)^{T}=\boldsymbol{J}_{22}^{-1} \boldsymbol{J}_{12}^{T}
$$

and, subsequently,

$$
\boldsymbol{J}_{12}^{T}-\boldsymbol{J}_{22} \boldsymbol{K}=\boldsymbol{J}_{12}^{T}-\boldsymbol{J}_{22} \boldsymbol{J}_{22}^{-1} \boldsymbol{J}_{12}^{T}=\mathbf{0}
$$

(48) implies

$$
\begin{aligned}
p_{w}(\boldsymbol{q}(i), \boldsymbol{w}(i)) & =p_{w}(\boldsymbol{w}(i)) \\
& =\boldsymbol{w}^{T}(i) \boldsymbol{W}^{T} \boldsymbol{J}_{22} \boldsymbol{W} \boldsymbol{w}(i) .
\end{aligned}
$$

Now, using the notation 47, (51) implies 45.

The value of Lemma 2 lies in the fact that in the forced regime the elements of the performance index sequence satisfy the basic zero condition (36) neither at time-varying states, nor at a steady state. On the other hand, (51) is independent of the state-feedback, and it can be considered a floating additive offset.

\subsection{Fault residual function.}

Definition 3. A quadratic function $h(\boldsymbol{q}(i), \boldsymbol{u}(i))$ is said to be a fault residual function if $h(\boldsymbol{q}(i), \boldsymbol{u}(i))=0$ for a linear deterministic discrete-time system without faults, and $h(\boldsymbol{q}(i), \boldsymbol{u}(i)) \neq 0$ in a fault regime of the system.

\section{Corollary 1.}

(i) For a steady-state solution $\boldsymbol{P}$ of (24) and the faultfree system (11), (2) in the unforced regime with the control policy (22), (23), the fault residual function $h(\boldsymbol{q}(i), \boldsymbol{u}(i))$ is

$$
h(\boldsymbol{q}(i), \boldsymbol{u}(i))=p(\boldsymbol{q}(i), \boldsymbol{u}(i))
$$

since (35) implies $p(\boldsymbol{q}(i), \boldsymbol{u}(i))=0$, and it represents the case when there is no fault in the system. That is why $p(\boldsymbol{q}(i), \boldsymbol{u}(i))$ can be used as the fault residual function.

(ii) For a steady-state solution $\boldsymbol{P}$ of (24) and the fault-free system (1), (2) in the forced regime with the control policy 
(22), (23), (40), the fault residual function $h(\boldsymbol{q}(i), \boldsymbol{u}(i))$ is

$$
h_{w}(\boldsymbol{q}(i), \boldsymbol{u}(i))=p(\boldsymbol{q}(i), \boldsymbol{u}(i))-p_{w}(\boldsymbol{w}(i))
$$

since the state-input relations given by (35), (51) allows defining (53). Thus, the steady-state value of $h_{w}(\boldsymbol{q}(i), \boldsymbol{u}(i))$ will be equal to zero if the deterministic closed-loop system is fault-free.

(iii) For a steady-state solution $\boldsymbol{P}$ of (24) denoting by $\boldsymbol{q}_{f}(t)$ the faulty system state vector, in the forced regime the control policy is

$$
\boldsymbol{u}_{f}(i)=-\boldsymbol{K} \boldsymbol{q}_{f}(i)+\boldsymbol{W} \boldsymbol{w}(i),
$$

where $\boldsymbol{K}$ and $\boldsymbol{W}$ are given as in (23) and (42), respectively. With respect to (53), this yields now

$$
\begin{aligned}
& h_{w}\left(\boldsymbol{q}_{f}(i), \boldsymbol{u}_{f}(i)\right) \\
& \quad=\left\|\boldsymbol{q}_{f}(i), \boldsymbol{u}_{f}(i)\right\|_{\boldsymbol{J}}^{2}-\|\boldsymbol{w}(i)\|_{\boldsymbol{W}^{\circ},}^{2}
\end{aligned}
$$

where

$$
\boldsymbol{W}^{\circ}=\boldsymbol{W}^{T} \boldsymbol{J}_{22} \boldsymbol{W} .
$$

Taking into account that $h_{w}(\boldsymbol{q}(i), \boldsymbol{u}(i))=0$ for the faultfree system, the fault residual function for the system in a fault regime takes the value

$$
\begin{aligned}
& h_{w}\left(\boldsymbol{q}_{f}(i), \boldsymbol{u}_{f}(i)\right) \\
& \quad=h_{w}\left(\boldsymbol{q}_{f}(i), \boldsymbol{u}_{f}(i)\right)-h_{w}(\boldsymbol{q}(i), \boldsymbol{u}(i)) \\
& \quad=\left\|\boldsymbol{q}_{f}(i), \boldsymbol{u}_{f}(i)\right\|_{\boldsymbol{J}}^{2}-\|\boldsymbol{q}(i), \boldsymbol{u}(i)\|_{\boldsymbol{J}}^{2} \neq 0 .
\end{aligned}
$$

where $\|\cdot\|_{\boldsymbol{X}}$ denotes the Mahalanobis norm of a vector with the positive definite weighting matrix $\boldsymbol{X}, \boldsymbol{J} \in$ $\mathbb{R}^{(n+r) \times(n+r}, \boldsymbol{J}=\boldsymbol{J}^{T}>0$ is given in (28).

(iv) For a steady-state solution $\boldsymbol{P}$ of (24) and the faulty system state vector $\boldsymbol{q}_{f}(t)$, in the unforced regime (54) gives

$$
\boldsymbol{u}_{f}(i)=-\boldsymbol{K} \boldsymbol{q}_{f}(i)
$$

and, consequently, (52) and (57) imply

$$
h_{w}\left(\boldsymbol{q}_{f}(i), \boldsymbol{u}_{f}(i)\right)=\left\|\boldsymbol{q}_{f}(i), \boldsymbol{u}_{f}(i)\right\|_{\boldsymbol{J}}^{2}>0 .
$$

Lemma 3. If the residual function takes the form (53), then an abrupt change in its response to a single sensor fault in the system is strengthened by $\boldsymbol{w}(i)$.

Proof. Considering a single sensor fault and neglecting sensor dynamics, the control feedback signal can be written as (Krokavec and Filasová, 2009)

$$
\begin{aligned}
\boldsymbol{u}_{f}(i) & =-\boldsymbol{K} \boldsymbol{q}_{f}(i)+\boldsymbol{W} \boldsymbol{w}(i) \\
& =-\boldsymbol{K}\left(\boldsymbol{I}-\boldsymbol{I}_{f s}\right) \boldsymbol{q}(i)+\boldsymbol{W} \boldsymbol{w}(i) \\
& =\boldsymbol{u}(i)+\boldsymbol{u}_{s}(i)
\end{aligned}
$$

where

$$
\boldsymbol{u}_{s}(i)=\boldsymbol{K} \boldsymbol{I}_{f s} \boldsymbol{q}(i)
$$

$\boldsymbol{u}(i)$ being defined as in 45, and $\boldsymbol{I}_{f s} \in \mathbb{R}^{n \times n}$ generally takes the form

$$
\boldsymbol{I}_{f s}=\operatorname{diag}\left[\begin{array}{lllllll}
0 & \ldots & 0 & 1 & 0 & \ldots & 0
\end{array}\right] .
$$

Since (53) can be expressed as

$$
\begin{aligned}
h_{w} & (\boldsymbol{q}(i), \boldsymbol{u}(i)) \\
= & \boldsymbol{q}^{T}(i) \boldsymbol{J}_{11} \boldsymbol{q}(i)+\boldsymbol{u}^{T}(i) \boldsymbol{J}_{22} \boldsymbol{u}(i) \\
& +2 \boldsymbol{q}^{T}(i) \boldsymbol{J}_{12} \boldsymbol{u}(i)-\boldsymbol{w}^{T}(i) \boldsymbol{W}^{T} \boldsymbol{J}_{22} \boldsymbol{W} \boldsymbol{w}(i),
\end{aligned}
$$

substituting $\boldsymbol{u}(i) \leftarrow \boldsymbol{u}_{f}(i)$ and $\boldsymbol{q}(i) \leftarrow \boldsymbol{q}_{f}(i)$ in (63) results in

$$
\begin{aligned}
h_{w}\left(\boldsymbol{q}_{f}(i), \boldsymbol{u}_{f}(i)\right) \\
=\boldsymbol{q}_{f}^{T}(i) \boldsymbol{J}_{11} \boldsymbol{q}_{f}(i)+2 \boldsymbol{q}_{f}^{T}(i) \boldsymbol{J}_{12} \boldsymbol{u}(i) \\
\quad+\boldsymbol{u}^{T}(i) \boldsymbol{J}_{22} \boldsymbol{u}(i)-\boldsymbol{w}^{T}(i) \boldsymbol{W}^{T} \boldsymbol{J}_{22} \boldsymbol{W} \boldsymbol{w}(i) \\
\quad+2\left(\boldsymbol{q}_{f}^{T}(i) \boldsymbol{J}_{12}+\boldsymbol{u}^{T}(i) \boldsymbol{J}_{22}\right) \boldsymbol{u}_{s}(i)+\boldsymbol{u}_{s}^{T}(i) \boldsymbol{J}_{22} \boldsymbol{u}_{s}(i), \\
h_{w}\left(\boldsymbol{q}_{f}(i), \boldsymbol{u}_{f}(i)\right) \\
=h_{w}\left(\boldsymbol{q}_{f}(i), \boldsymbol{u}(i)\right) \\
\quad+2\left(\boldsymbol{q}_{f}^{T}(i) \boldsymbol{J}_{12}+\boldsymbol{u}^{T}(i) \boldsymbol{J}_{22}\right) \boldsymbol{u}_{s}(i) \\
\quad+\boldsymbol{u}_{s}^{T}(i) \boldsymbol{J}_{22} \boldsymbol{u}_{s}(i)-\boldsymbol{w}^{T}(i) \boldsymbol{W}^{T} \boldsymbol{J}_{22} \boldsymbol{W} \boldsymbol{w}(i),
\end{aligned}
$$

respectively, where

$$
\begin{aligned}
h_{w}\left(\boldsymbol{q}_{f}(i), \boldsymbol{u}(i)\right) \\
=2 \boldsymbol{q}_{f}^{T}(i) \boldsymbol{J}_{12} \boldsymbol{u}(i) \\
\quad+\boldsymbol{q}_{f}^{T}(i) \boldsymbol{J}_{11} \boldsymbol{q}_{f}(i)+\boldsymbol{u}^{T}(i) \boldsymbol{J}_{22} \boldsymbol{u}(i) .
\end{aligned}
$$

Now, using (60), i.e., $\boldsymbol{u}(i)=-\boldsymbol{u}_{s}(i)-\boldsymbol{K} \boldsymbol{q}_{f}(i)+$ $\boldsymbol{W} \boldsymbol{w}(i)$, we get

$$
\begin{aligned}
& h_{w}\left(\boldsymbol{q}_{f}(i), \boldsymbol{u}_{s}(i)\right) \\
& =2\left(\boldsymbol{q}_{f}^{T}(i) \boldsymbol{J}_{12}+\boldsymbol{u}^{T}(i) \boldsymbol{J}_{22}\right) \boldsymbol{u}_{s}(i) \\
& \quad+\boldsymbol{u}_{s}^{T}(i) \boldsymbol{J}_{22} \boldsymbol{u}_{s}(i)-\boldsymbol{w}^{T}(i) \boldsymbol{W}^{T} \boldsymbol{J}_{22} \boldsymbol{W} \boldsymbol{w}(i) \\
& =2 \boldsymbol{w}^{T}(i) \boldsymbol{W}^{T} \boldsymbol{J}_{22} \boldsymbol{u}_{s}(i) \\
& \quad+2 \boldsymbol{u}_{s}^{T}(i)\left(\boldsymbol{J}_{12}^{T}-\boldsymbol{J}_{22} \boldsymbol{K}\right) \boldsymbol{q}_{f}(i) \\
& \quad-\boldsymbol{u}_{s}^{T}(i) \boldsymbol{J}_{22} \boldsymbol{u}_{s}(i)-\boldsymbol{w}^{T}(i) \boldsymbol{W}^{T} \boldsymbol{J}_{22} \boldsymbol{W} \boldsymbol{w}(i),
\end{aligned}
$$

and it is clear that, with respect to $50, h_{w}\left(\boldsymbol{q}_{f}(i), \boldsymbol{u}_{s}(i)\right)$ takes the form

$$
\begin{aligned}
h_{w}\left(\boldsymbol{q}_{f}(i), \boldsymbol{u}_{s}(i)\right)= & h_{w}\left(\boldsymbol{u}_{s}(i)\right) \\
= & 2 \boldsymbol{w}^{T}(i) \boldsymbol{W}^{T} \boldsymbol{J}_{22} \boldsymbol{u}_{s}(i)-\boldsymbol{u}_{s}^{T}(i) \boldsymbol{J}_{22} \boldsymbol{u}_{s}(i) \\
& -\boldsymbol{w}^{T}(i) \boldsymbol{W}^{T} \boldsymbol{J}_{22} \boldsymbol{W} \boldsymbol{w}(i) .
\end{aligned}
$$




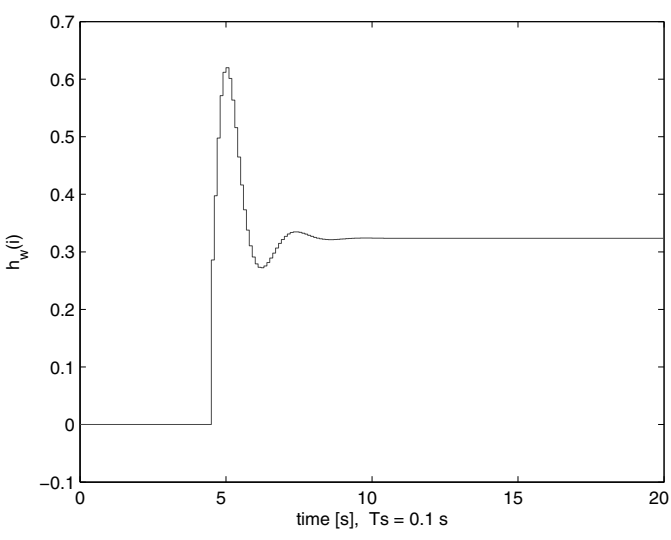

(a)

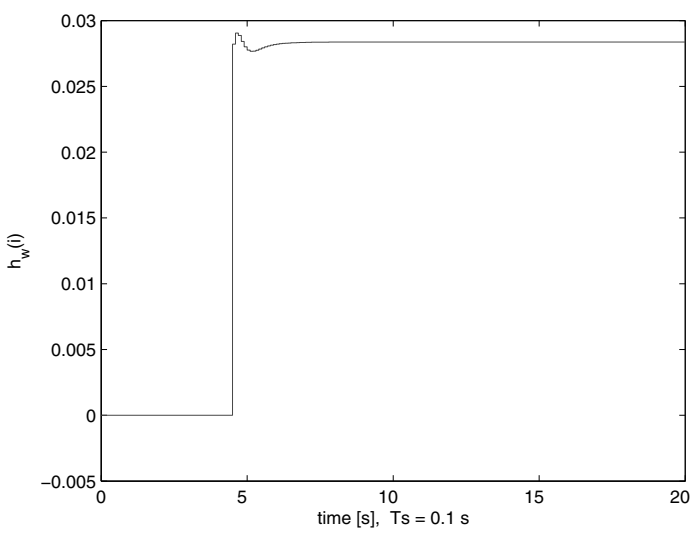

(b)

Fig. 1. Responses of the fault residual function (53): the second sensor fault (a), the third sensor fault (b).

Further, respecting the structure of (17), $h_{w}\left(\boldsymbol{u}_{s}(i)\right)$ can be expressed in a concise form,

$$
h_{w}\left(\boldsymbol{u}_{s}(i)\right)=\left[\begin{array}{ll}
\boldsymbol{w}^{T}(i) & \boldsymbol{u}_{s}^{T}(i)
\end{array}\right] \boldsymbol{W}_{s}\left[\begin{array}{c}
\boldsymbol{w}(i) \\
\boldsymbol{u}_{s}(i)
\end{array}\right],
$$

where

$$
\boldsymbol{W}_{s}=\left[\begin{array}{cc}
-\boldsymbol{W}^{T} \boldsymbol{J}_{22} \boldsymbol{W} & \boldsymbol{W}^{T} \boldsymbol{J}_{22} \\
\boldsymbol{J}_{22} \boldsymbol{W} & -\boldsymbol{J}_{22}
\end{array}\right] .
$$

Since $\boldsymbol{J}_{22}$ is positive definite, applying Schur's complement property to (70) will yield

$$
-\boldsymbol{W}^{T} \boldsymbol{J}_{22} \boldsymbol{W}+\boldsymbol{W}^{T} \boldsymbol{J}_{22} \boldsymbol{W}=0 \Leftrightarrow \boldsymbol{W}_{s} \geq 0,
$$

which implies that $h_{w}\left(\boldsymbol{u}_{s}(i)\right)$ is positive semi-definite.

3.4. Responses of residual functions. Principally, using LQ control, there may exist some faults which do not cause instability of the closed-loop system, but their permanent occurrence is technically unacceptable. The above introduced fault residual function (53) represents a constructive procedure for detecting faults that cause system instability, as well as for detecting single sensor faults against which the control system is robust, i.e., the responses of the fault residual function (53) are unbounded in the presence of faults which give rise to closed-loop system instability, nonzero but bounded in the presence of single sensor faults against which the control system is robust, and are approximately zero in the presence of other faults against which the controlled system is robust.

To detect faults against which the control system is robust, with the exception of sensor faults of this kind, another form of the modified fault residual function is proposed,

$$
\begin{aligned}
h_{w} & (\boldsymbol{q}(i+1), \boldsymbol{q}(i), \boldsymbol{u}(i)) \\
= & \boldsymbol{q}^{T}(i+1) \boldsymbol{Y} \boldsymbol{q}(i+1)-\boldsymbol{q}^{T}(i) \boldsymbol{Y} \boldsymbol{q}(i) \\
& +\boldsymbol{q}^{T}(i) \boldsymbol{Q} \boldsymbol{q}(i)+2 \boldsymbol{q}^{T}(i) \boldsymbol{S} \boldsymbol{u}(i)+\boldsymbol{u}^{T}(i) \boldsymbol{R} \boldsymbol{q}(i) \\
& -\boldsymbol{w}^{T}(i) \boldsymbol{W}^{T}\left(\boldsymbol{G}^{T} \boldsymbol{P} \boldsymbol{G}+\boldsymbol{R}\right) \boldsymbol{W} \boldsymbol{w}(i),
\end{aligned}
$$

changing the dynamics of the fault residual function, where $\boldsymbol{Y} \in \mathbb{R}^{n \times n}$ is an arbitrary real symmetric positive definite matrix. It is obvious that in a steady-state, where $\boldsymbol{q}(i+1)=\boldsymbol{q}(i)$, the modified $h_{w}(\boldsymbol{q}(i+1), \boldsymbol{q}(i), \boldsymbol{u}(i))$ is equal to $h_{w}(\boldsymbol{q}(i), \boldsymbol{u}(i))$.

Note that this modification does not significantly change the evaluation procedure with respect to faults in the controlled system. It is evident that for a stochastic noisy system a threshold setting is necessary.

\section{Illustrative examples}

Illustrations of the proposed scheme and the effectiveness of the algorithm are presented using the model suitable for fault tolerant control system verification (Krokavec and Filasová, 2009). The matrix parameters of the system are

$$
\begin{gathered}
\boldsymbol{F}=\left[\begin{array}{rrr}
0.9993 & 0.0987 & 0.0042 \\
-0.0212 & 0.9612 & 0.0775 \\
-0.3875 & -0.7187 & 0.5737
\end{array}\right], \\
\boldsymbol{G}=\left[\begin{array}{rr}
0.1101 & 0.3056 \\
0.2008 & 0.1176 \\
-0.0213 & 0.2853
\end{array}\right], \quad \boldsymbol{C}=\left[\begin{array}{rrr}
1 & 2 & -2 \\
1 & -1 & 0
\end{array}\right]
\end{gathered}
$$

for the sampling period $\Delta t=0.1 \mathrm{~s}$. The system is in closed loop under the state feedback (40) where the control law gain matrix

$$
\boldsymbol{K}=\left[\begin{array}{rrr}
0.4160 & 1.0763 & -0.0248 \\
0.8798 & 0.3686 & 0.0971
\end{array}\right]
$$




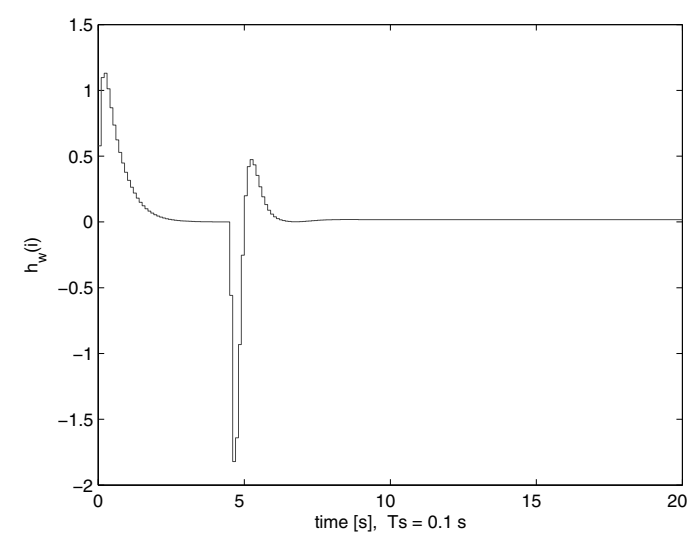

(a)

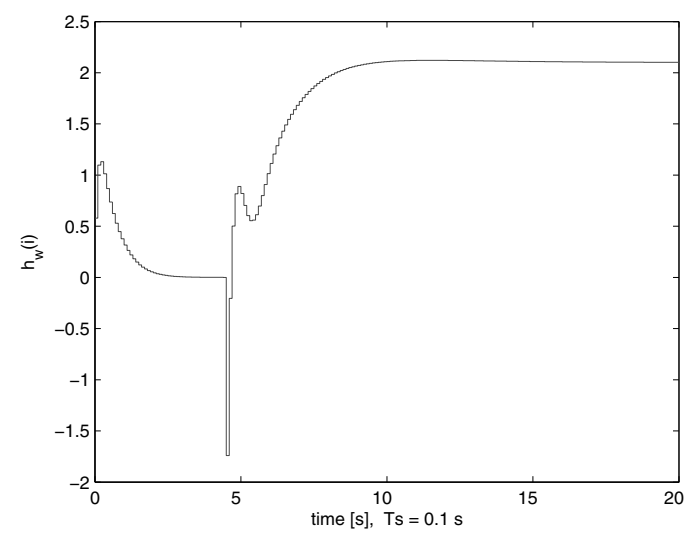

(b)

Fig. 2. Responses of the fault residual function (72): the first actuator fault (a), the second actuator fault (b).

was directly synthesized using the standard Matlab function dare $(F, G, Q, R, S, E)$.

Note that dare solves a more general task and computes a unique stabilizing solution $\boldsymbol{P}$ of the discrete-time algebraic Riccati equation of the form

$$
\begin{array}{rl}
\boldsymbol{E} & \boldsymbol{P} \boldsymbol{E} \\
= & \boldsymbol{F}^{T} \boldsymbol{P} \boldsymbol{F}+\boldsymbol{Q}\left(\boldsymbol{F}^{T} \boldsymbol{P} \boldsymbol{G}+\boldsymbol{S}\right)\left(\boldsymbol{G}^{T} \boldsymbol{P} \boldsymbol{G}+\boldsymbol{R}\right)^{-1} \\
& \times\left(\boldsymbol{F}^{T} \boldsymbol{P} \boldsymbol{G}+\boldsymbol{S}\right)^{T}
\end{array}
$$

Compared with (24), it is clear that the parameter matrix $\boldsymbol{E}$ has to be equal to the identity matrix $\boldsymbol{I}_{n}$. For more details, see, e.g., the work of Lancaster and Rodman (1995).

It is possible to verify that the first sensor fault, as well as the change in the system matrix parameter $F_{22}$ to 1.3457 gives rise to closed-loop system instability, while LQ control is robust with respect to single actuator faults, to faults of the second and the third sensor, as well as to a change in the system matrix parameter $F_{22}$ from $[0.9612,1.1534]$.

To adjust the lower-bound of supply rate, as well as (reflecting Bryson's rule) to set an identical constraint on the system state and output variables, the performance index matrices were determined using the SeDuMi package for Matlab, as a solution of the linear matrix inequality 12, i.e.,

$$
\begin{gathered}
Q=0.71 \boldsymbol{I}_{3}, \quad \boldsymbol{R}=0.71 \boldsymbol{I}_{2}, \\
\boldsymbol{S}^{T}=10^{-7}\left[\begin{array}{rrr}
0.0006 & -0.0000 & 0.1603 \\
0.1727 & 0.0000 & -0.0007
\end{array}\right] .
\end{gathered}
$$

In this sense, the cross coupling weight $S$ can be interpreted as a matrix concerning the lower bound of the supply rate in LQ control.
Thus, the following matrices were produced directly:

$$
\begin{gathered}
\boldsymbol{P}=\left[\begin{array}{rrr}
4.6184 & 0.7659 & -0.6462 \\
0.7659 & 5.2336 & -0.3005 \\
-0.6462 & -0.3005 & 1.1445
\end{array}\right], \\
\boldsymbol{W}=\left[\begin{array}{rr}
0.3889 & -0.1393 \\
-0.0175 & 0.6124
\end{array}\right], \quad \boldsymbol{Y}=20 \boldsymbol{Q},
\end{gathered}
$$

the system initial state vector was $\boldsymbol{q}(0)=\mathbf{0}$, and the desired input signal vector $\boldsymbol{w}(i)$ was adjusted so that

$$
\boldsymbol{w}^{T}(i)=\left[\begin{array}{ll}
1 & -0.5
\end{array}\right] \text {. }
$$

In simulations there we considered faults which did not cause closed-loop system instability, modeled by step functions starting at any time instant in a system steady state. Thus, Fig. 1 represents fault residual function responses, as the output of (53) reflecting a single fault of the second and the third sensor, respectively, starting at the time instant $t=4.5 \mathrm{~s}$. Figure 2 presents the responses of the fault residual function (72) in analogous situations concerning the single actuator faults, and Fig. 3 illustrates the response of the same residual function with respect to the step change in the nominal parameter $F_{22}$ up to a value of 1.1534, all starting at the time instant $t=4.5 \mathrm{~s}$.

\section{Concluding remarks}

The fault detection scheme described in the paper is based on evaluating LQ control performance index parameters, where the faults influences are analyzed with respect to the fault residual functions $h_{w}(\boldsymbol{q}(i), \boldsymbol{u}(i))$ and $h_{w}(\boldsymbol{q}(i+1), \boldsymbol{q}(i), \boldsymbol{u}(i))$ under the assumption of full state feedback control. In addition, analyzing effects associated with the faults, the fault residual function responses allow separating a class of single sensor faults and a class of other faults, whose occurrence does not cause 


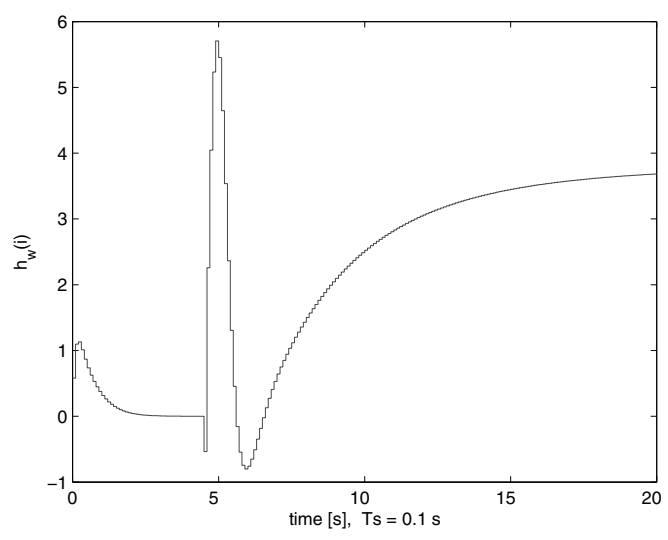

Fig. 3. Response of the residual function 72 : the robust system parameter $F_{22}$ fault.

instability of the closed-loop system. Since the fault detection scheme extracts fault distribution information from controller input and output sequences only and does not exploit the system dynamics and structure in any way (e.g., by means of a system model), it evidently does not allow a complete fault isolation. On the other hand, the proposed recursive algorithms are parameterized only by the controller design parameters $Q, R, S$, and can be potentially extended to be implementable, e.g., in simple embedded diagnostic structures for SISO systems, or combined with other principles which allow fault isolation.

The proposed method presents some new design features and generalizations, and it is emphasized that the advantage offered by such an approach is a simple recursive algorithm that does not need extra dynamics. Since the application is conditioned by an LQ control realization, from the point of view of investigating a fault detection system design, it can constitute a first step in developing a practical method based on LQ control performance index evaluation. The next step in the development of the proposed scheme is first the extension to full state unmeasurable linear systems and linear-parameter varying systems.

\section{Acknowledgment}

The work presented in the paper was supported by VEGA, the Grant Agency of the Ministry of Education and the Academy of Sciences of the Slovak Republic, under Grant No. 1/0256/11. This support is very gratefully acknowledged. The authors would also like to thank the anonymous reviewers for their detailed constructive comments and suggestions, which helped to improve the paper.

\section{References}

Anderson, B.D.O. and Moore, J.B. (1989). Optimal Control. Linear Quadratic Methods, Prentice-Hall, Englewood Cliffs, NJ.

Blanke, M., Kinnaert, M., Lunze, J. and Staroswiecki, M. (2003). Diagnosis and Fault-Tolerant Control, Springer-Verlag, Berlin.

Brogliato, B., Lozano, R., Maschke, B. and Egeland, O. (2007). Dissipative Systems Analysis and Control. Theory and Applications, Springer-Verlag, London.

Bryson A.E. (2002). Applied Linear Optimal Control. Examples and Algorithms, Cambridge University Press, Cambridge.

Bryson A.E. and Ho, Y.C. (1975). Applied Optimal Control. Optimization, Estimation, and Control, Taylor \& Francis, New York, NY.

Chen, J. and Patton, R.J. (1999). Robust Model-Based Fault Diagnosis for Dynamic Systems, Kluwer, Norwell, MA.

Chen, W., Ding, S.X., Khan, A.Q. and Abid. M. (2010). Energy based fault detection for dissipative systems, Conference on Control and Fault Tolerant Systems, SysTol 2010, Nice, France, pp. 517-521.

Chen, W., Khan, A.Q., Abid. M. and Ding, S.X., (2011). Integrated design of observer based fault detection for a class of uncertain nonlinear systems, International Journal of Applied Mathematics and Computer Science 21(3): 423-430, DOI: 10.2478/v10006-011-0031-0.

Ding, S.X. (2009). Integrated design of control structures and embedded diagnosis, 7th IFAC International Symposium on Fault Detection, Supervision and Safety of Technical Processes, SAFEPROCESS 2009, Barcelona, Spain, pp. 734-745.

Dorf, R.C. and Bishop, R.H. (2011). Modern Control Systems, Pearson Education, Upper Saddle River, NJ.

Furuta, K. and Kim, S.B. ( 1987) Pole assignment in a specified disk, IEEE Transactions on Automatic Control 32(5): 423-427.

Haddad, W.M. and Chellaboina, V.S. (2003). Nonlinear Dynamical Systems and Control. A Lyapunov-Based Approach, Princeton University Press, Princeton, NJ.

Hendricks, E., Jannerup, O. and Sørensen P.H. (2008). Linear Systems Control. Deterministic and Stochastic Methods, Springer-Verlag, Berlin.

Henry, D. (2010). A norm-based point of view for fault diagnosis. Application to aerospace missions, 8th European Workshop on Advanced Control and Diagnosis, Ferrara, Italy, pp. 4-16.

Kirk, D.E. (1970). Optimal Control Theory. An Introduction, Prentice Hall, Englewood Cliffs, NJ.

Kailath, T. (1980). Linear Systems, Prentice-Hall, Englewood Cliffs, NJ.

Khalil, H.K. (2002). Nonlinear Systems, Prentice Hall, Upper Saddle River, NJ. 
Khelassi, A., Theilliol, D. and Weber, P. (2011). Reconfigurability analysis for reliable fault tolerant control design, International Journal of Applied Mathematics and Computer Science 21(3): 431-439, DOI: 10.2478/v10006-011-0032-z.

Korbicz, J., Kościelny, J. M., Kowalczuk, Z. and Cholewa, W. (Eds.) (2004). Fault Diagnosis. Models, Artificial Intelligence, Applications, Springer-Verlag, Berlin.

Korbicz, J. (2007). Fault diagnosis of non-linear dynamical systems using analytical and soft computing methods, Journal of Automation, Mobile Robotics \& Intelligent Systems, 1(1): 7-23.

Krokavec, D. (2002). Convergence of action dependent dual heuristic dynamic programming algorithms in LQ control tasks, Intelligent Technologies-Theory and Application. New Trends in Intelligent Technologies, IOS Press, Amsterdam, pp. 72-80.

Krokavec, D. and Filasová, A. (2008). Discrete-Time Systems, Elfa, Košice, (in Slovak).

Krokavec, D. and Filasová, A. (2009). Control reconfiguration based on the constrained LQ control algorithms, 7th IFAC International Symposium on Fault Detection, Supervision and Safety of Technical Processes, SAFEPROCESS 2009, Barcelona, Spain, pp. 686-691.

Lancaster, P. and Rodman, L. (1995). Algebraic Riccati Equations, Clarendon Press, Oxford.

Lewis, F.L. and Syrmos, V.L. (1995) Optimal Control, John Wiley \& Sons, New York, NY.

Naidu, D.S. (2003). Optimal Control Systems, CRC Press, Boca Raton, FL.

Muhafzan (2010). Use of semidefinite programming for solving the LQR problem subject to rectangular descriptor systems, International Journal of Applied Mathematics and Computer Science 20(4): 655-664, DOI: 10.2478/v10006-010-0048-9.

Simani, S., Fantuzzi, C. and Patton, R.J. (2003). Model-Based Fault Diagnosis in Dynamic Systems Using Identification Techniques, Springer-Verlag, London.
Zolghadri, A. (2000). A redundancy-based strategy for safety management in a modern civil aircraft, Control Engineering Practice 8(5): 545-554.

Zolghadri, A., Castang, F., Henry, D. (2006). Design of robust fault detection filters for multivariable feedback systems, International Journal of Modeling and Simulation 26(1): $17-26$.

Dušan Krokavec received an M.Sc. degree in automatic control in 1967 and a Ph.D. degree in technical cybernetics in 1982 from the Faculty of Electrical Engineering, Slovak University of Technology in Bratislava, Slovakia. In 1984 he was promoted to an associated professor at the Technical University in Košice, Slovakia, and in 1999 he was appointed a full professor of automation and control. From 1968 to 1971 he was a research assistant at the Research Institute of Automation and Mechanization in Nové Mesto n/Váhom, Slovakia, and since 1971 he has been with the Department of Cybernetics and Artificial Intelligence, Faculty of Electrical Engineering and Informatics, Technical University of Košice. In the long term, he specializes in stochastic processes in dynamic systems, digital control systems and digital signal processing, and in dynamic system fault diagnosis. Professor Krokavec is a member of the IFAC Technical Committee on Stochastic Systems.

Anna Filasová graduated in technical cybernetics and received an M.Sc. degree in 1975 and a Ph.D. degree in 1993, both from the Faculty of Electrical Engineering and Informatics, Technical University of Košice, Slovakia. In 1999 she was appointed an associate professor at the Technical University in Košice. She is with the Department of Cybernetics and Artificial Intelligence, Faculty of Electrical Engineering and Informatics, and she worked there as an assistant professor from 1975 to 1999. Her main research interests are in robust and predictive control, decentralized control, large-scale system optimization, and control reconfiguration.

Received: 7 July 2011

Revised: 17 February 2012 